\title{
Manipulation of the Coronavirus Genome Using Targeted RNA Recombination with Interspecies Chimeric Coronaviruses
}

\author{
Cornelis A.M. de Haan, Bert Jan Haijema, Paul S. Masters, \\ and Peter J.M. Rottier
}

\begin{abstract}
Targeted RNA recombination has proven to be a powerful tool for the genetic engineering of the coronavirus genome, particularly in its $3^{\prime}$ part. Here we describe procedures for the generation of recombinant and mutant mouse hepatitis virus and feline infectious peritonitis virus. Key to the two-step method is the efficient selection of recombinant viruses based on host cell switching. The first step consists of the preparation-using this selection principle - of an interspecies chimeric coronavirus. In this virus the ectodomain of the spike glycoprotein is replaced by that of a coronavirus with a different species tropism. In the second step this chimeric virus is used as the recipient for recombination with synthetic donor RNA carrying the original spike gene. Recombinant viruses are then isolated on the basis of their regained natural (e.g., murine or feline) cell tropism. Additional mutations created in the donor RNA can be co-incorporated into the recombinant virus in order to generate mutant viruses.
\end{abstract}

Key words: coronavirus; mouse hepatitis virus; feline infectious peritonitis virus; reverse genetics; targeted RNA recombination; host cell switching; tropism

\section{Introduction}

Targeted RNA recombination was the first reverse genetics method developed to introduce mutations into the coronavirus genome [for a recent review, see (1)]. Actually, its first demonstration involved the repair of an 87-nucleotide deletion

From: Methods in Molecular Biology, vol. 454: SARS- and Other Coronaviruses,

Edited by: D. Cavanagh, DOI: 10.1007/978-1-59745-181-9_17, C Humana Press, New York, NY 
in the $\mathrm{N}$ gene of a temperature-sensitive mutant of the mouse hepatitis virus (MHV) by recombination with a synthetic donor RNA equivalent to the subgenomic mRNA for the wild-type $\mathrm{N}$ gene (2). Recombinant virus was selected by its increased temperature stability and efficient growth at the nonpermissive temperature, a tedious process. The frequency of RNA recombination and hence the ease with which recombinant viruses could be isolated was subsequently improved by the use of defective interfering (DI) RNAs as donors, i.e., RNA constructs consisting of sequences derived from the $5^{\prime}$ and $3^{\prime}$ terminal parts of the MHV genome $(3,4)$. Yet, selection of mutant viruses remained the Achilles' heel of the method, particularly when one was seeking to construct temperaturesensitive or otherwise crippled mutants. Hence, the approach did not become common practice.

A decisive improvement emerged from studies on the assembly of the spike into the coronavirion. With the use of virus-like particles it was shown that this process is governed by the spike (S) protein's carboxy-terminal region, comprising the transmembrane and cytoplasmic domains (5). Spikes were still incorporated into virus-like particles after replacement of their ectodomain by that of another coronavirus. Such chimeric spikes also retained their biological functions - receptor binding and membrane fusion — as judged by their ability to cause cell-cell fusion. Given the generally strict species-specific nature of coronaviruses in tissue culture these features enabled the design of a powerful positive selection strategy based on interspecies chimeric coronaviruses, as was first described for MHV (6).

Here we describe the detailed procedures for the method of targeted RNA recombination using this strategy of host cell switching. Protocols are presented for MHV and FIPV (feline infectious peritonitis virus) as examples. For any given virus the procedure starts with the generation of an interspecies chimeric coronavirus. Thus, a donor DI RNA is prepared containing a chimeric $\mathrm{S}$ gene. Cells are infected with the virus in question and additionally transfected with the donor RNA. In such cells homologous recombination of the donor RNA with viral genomic RNA can give rise to the formation and release of chimeric virus. This chimeric virus is subsequently selected by passing the culture supernatant onto cells that are infectable only by the virus from which the chimeric $\mathrm{S}$ protein ectodomain was derived. Felinized MHV (fMHV) and murinized FIPV (mFIPV) are thus the chimeric viruses described in the examples; they are isolated and grown in feline and murine cells, respectively. The chimeric viruses subsequently function as the recipients for the second round of homologous recombination designed to regenerate the original virus or to obtain mutants thereof. Donor DI RNA is again prepared that now contains the original wildtype $\mathrm{S}$ gene, plus any additional intended mutations. In cells infected with the chimeric virus, recombination with this RNA can lead to formation of (mutants 


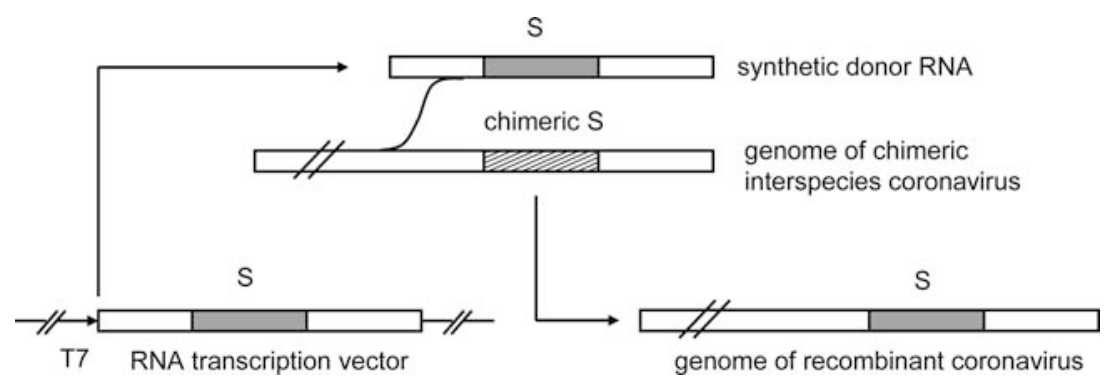

Fig. 1. Second round of homologous recombination to regenerate the original virus or to obtain mutants thereof. Donor DI RNA with the original wild-type S gene plus any additional intended mutations are introduced into cells that have been infected with the chimeric virus. Recombination with this RNA can lead to formation of (mutants of) the original virus. These are isolated by passing the collection of progeny viruses onto its natural cells.

of) the original virus, which can now, in turn, be isolated by passing the collection of progeny viruses onto its natural cells. A schematic outline of the latter recombination process is depicted in Fig. 1.

Owing to restrictions inherent in the selection principle, the targeted RNA recombination system described here will have its main value in the study and manipulation of functions specified by the genomic regions downstream of the polymerase gene. For a survey of important contributions that the targeted recombinant approach has made in the different areas of its application, the reader is referred to the review by Masters and Rottier (1).

\section{Materials}

\subsection{Plasmids and RNA Synthesis}

1. Plasmids pMH54 (6) and pBRDI1 (7) are used for the generation of MHV and FIPV recombinants, respectively.

2. Restriction enzymes Pac I and Not I (New England Biolabs) are used in combination with the supplied reaction buffer.

3. $3 \mathrm{M}$ ammonium acetate solution (see Note 1).

4. Ethanol $70 \%(\mathrm{v} / \mathrm{v})$.

5. Ethanol $96 \%(\mathrm{v} / \mathrm{v})$.

6. T7 RNA polymerase kit (Ambion).

7. Agarose (Invitrogen).

8. 1X Tris-borate electrophoresis buffer (TBE): 0.09 M Tris-borate, 0.002 M EDTA. $0.5 \mathrm{X}$ Tris-acetate electrophoresis buffer (TAE): $0.02 \mathrm{M}$ Tris-acetate, $0.0005 \mathrm{EDTA}$. 


\subsection{Cell Culture and Plaque Assay}

1. Dulbecco's Modified Eagle's Medium (DMEM; Biowhittaker) supplemented with $10 \%$ fetal bovine serum (FBS; HyClone), $100 \mathrm{IU}$ of penicillin/ml and $100 \mu \mathrm{g}$ of streptomycin/ml (p/s) (DMEM++).

2. Both murine LR7 cells (5) and feline FCWF cells (ATCC) are used for the generation of MHV and FIPV recombinants (see Note 2).

3. Solutions of trypsin (500 mg/liter trypsin 1:250) and EDTA (200 mg/liter) (Trypsin EDTA solution; Biowhittaker).

4. Dulbecco's phosphate buffered saline (DPBS) without $\mathrm{Ca}$ and $\mathrm{Mg}$.

5. Select agar (Invitrogen).

6. 2X Minimal Essential Medium Eagle (EMEM; Biowhittaker) supplemented with $20 \% \mathrm{FBS}$ and $2 \mathrm{X} \mathrm{p} / \mathrm{s}$.

\subsection{Infection and Electroporation}

1. Recipient viruses fMHV and mFIPV are used for the generation of MHV and 2. FIPV recombinants, respectively.

2. 0.4-cm Gene Pulser cuvette (Biorad).

3. Biorad Gene Pulser II apparatus with Capacitance extender plus.

\section{Methods}

The procedures for the two steps of the method-preparation of the chimeric recipient virus and re-creation of the original virus (with or without additional mutations) - are identical, except for the reciprocal way the recombinant viruses are selected. Hence, we have chosen to describe here the protocols for the second step only, i.e., for generating recombinant forms of wild-type MHV and FIPV; the chimeric viruses fMHV and mFIPV are assumed to have been prepared similarly.

For the preparation of recombinant MHV and FIPV, we used, respectively, RNA transcription vectors pMH54 and pBRDI1. The generation of these plasmids has been described in detail by Kuo et al. (6) and by Haijema and co-workers (7), respectively. pMH54 specifies a defective MHV-A59 RNA transcript consisting of the 5'-end of the genome (467 nt) fused to codon 28 of the $\mathrm{HE}$ gene and running to the 3'-end of the genome; pBRDI1 specifies a similar FIPV transcript.

Both viral cDNA constructs are cloned behind a bacteriophage T7 RNA polymerase promoter. Mutations to be created in the MHV or FIPV genomes are introduced into these cDNA constructs in order to be carried through the transcribed RNA into the recombinant virus.

For the generation of recombinant MHV, feline FCWF cells are infected with fMHV, after which donor RNA transcribed from pMH54 is transfected by 
electroporation. Next, the recombinant MHV is selected on murine LR7 cells. For the generation of recombinant FIPV, the LR7 cells are infected with mFIPV, after which the donor RNA transcribed from pBRDI1 is transfected by electroporation. Subsequently, the recombinant FIPV is selected on FCWF cells.

\subsection{Preparation of Recipient Virus Stocks}

1. Maintain the LR7 and FCWF cells by regular passaging, using Trypsin EDTA solution when the cells approach confluency, to provide new maintenance cultures as well as cultures for the generation of the recipient virus stocks.

2. A 1:15 split of LR7 cells and a 1:3 split of FCWF cells will usually provide cultures that approach confluence after $72 \mathrm{~h}$.

3. Wash LR7 or FCWF cells once with DMEM before inoculating with mFIPV or fMHV, respectively, in DMEM at a multiplicity of infection (MOI) of 0.05-0.1 $\mathrm{TCID}_{50}$ (50\% tissue culture infectious doses) per cell (see Note 2).

4. After $1 \mathrm{~h}$ incubation at $37^{\circ} \mathrm{C}$, remove the inoculum and wash the cells once with DMEM, and then overlay them with DMEM++.

5. Harvest the culture medium containing the viruses typically after $24 \mathrm{~h}$ incubation at $37^{\circ} \mathrm{C}$, when the monolayers exhibit extensive cytopathic effects and detach from the plastic.

6. Transfer the medium to $50-\mathrm{ml}$ polypropylene tubes and clarify by centrifugation for $10 \mathrm{~min}$ at $2500 \mathrm{rpm}$ at room temperature using a Labofuge GL (Heraeus) or a comparable centrifuge.

7. Store the supernatant in aliquots at $-80^{\circ} \mathrm{C}$ until use. Typical titers obtained for fMHV and $\mathrm{mFIPV}$ are around $10^{6} \mathrm{TCID}_{50} / \mathrm{ml}$.

\subsection{Preparation of Synthetic Donor RNA}

1. Linearize $10 \mu \mathrm{g}$ of pMH54 or pBRDI1 with $30 \mathrm{U}$ Pac I or Not I restriction enzyme, respectively, using the reaction buffers supplied by New England Biolabs in a reaction volume of $100 \mu \mathrm{l}$ for $2 \mathrm{~h}$ at $37^{\circ} \mathrm{C}$.

2. Check the linearization of the plasmids by analyzing an aliquot of the reaction mixture using $1 \%(\mathrm{w} / \mathrm{v})$ agarose gel electrophoresis in $0.5 \mathrm{X}$ TAE.

3. Precipitate the linearized plasmid DNA by addition of one-tenth volume of $3 \mathrm{M}$ ammonium acetate and 2.5 volumes of $96 \%(\mathrm{v} / \mathrm{v})$ ethanol, followed by incubation at $-20^{\circ} \mathrm{C}$ for $30 \mathrm{~min}$.

4. Pellet the DNA precipitates using an Eppendorf centrifuge (13,000 rpm, $15 \mathrm{~min}$, $\left.4^{\circ} \mathrm{C}\right)$.

5. Wash the pellet with $70 \%(\mathrm{v} / \mathrm{v})$ ethanol, dry it, and take it up in $10 \mu \mathrm{l}$ of water (see Note 3).

6. Transcribe synthetic RNA in a $20-\mu 1$ reaction volume, using the T7 Message Machine kit (Ambion) according to the manufacturer's instructions, with the following modifications. 
a. Instead of $1 \mu \mathrm{g}$ of DNA, $3 \mu \mathrm{l}$ of the concentrated, linearized plasmid DNA is used.

b. In addition, $3 \mu \mathrm{l}$ of $30 \mathrm{mM}$ GTP (present in the kit) is added, to enhance transcription of large RNAs (see Note 4).

7. Check RNA synthesis by analyzing one-tenth of the reaction volume by electrophoresis in a $1 \%(\mathrm{w} / \mathrm{v})$ agarose gel in $1 \mathrm{X}$ TBE.

8. Store the RNA at $-80^{\circ} \mathrm{C}$ until use.

\subsection{Infection and Electroporation}

1. Typically, one $80-\mathrm{cm}^{2}$ flask containing a culture of cells reaching confluence is sufficient for two RNA electroporations.

2. Infect FCWF or LR7 cells with the recipient viruses fMHV or mFIPV, respectively, at an MOI of $0.5-1 \mathrm{TCID}_{50} /$ cell in DMEM++.

3. After $4 \mathrm{~h}$ of incubation at $37^{\circ} \mathrm{C}$, detach the cells from the plate using Trypsin EDTA solution $\left(1.5 \mathrm{ml}\right.$ per $\left.80 \mathrm{~cm}^{2}\right)$.

4. Add approximately $10 \mathrm{ml}$ DMEM++ to the cell suspension.

5. Pellet the cells by centrifugation in 50-ml polypropylene tubes for $10 \mathrm{~min}$ at $500 \mathrm{rpm}$ at room temperature.

6. Remove the supernatant and gently take up the pellet in DPBS without Ca and $\mathrm{Mg}$.

7. Pellet the cells by centrifugation in $50-\mathrm{ml}$ polypropylene tubes for $10 \mathrm{~min}$ at $500 \mathrm{rpm}$ at room temperature.

8. Remove the supernatant and gently take up the cell pellet in DPBS without Ca and $\mathrm{Mg}\left(1 \mathrm{ml} / 80 \mathrm{~cm}^{2}\right.$ original monolayer). Gently pipette the cell suspension carefully up and down until all cell clumps have disappeared.

9. Transfer $800 \mu \mathrm{l}$ of the cell suspension to a $0.4-\mathrm{cm}$ Gene Pulser cuvette containing $9 \mu \mathrm{l}$ of the synthetic donor RNA.

10. Mix the cells and RNA by pipetting gently.

11. Subject the RNA/cell mixture to two consecutive pulses using the Biorad Gene Pulser II with capacitance extender plus $(0.3 \mathrm{kV}, 950 \mu \mathrm{F})$.

12. Add the electroporated cell suspension to $8 \mathrm{ml} \mathrm{DMEM}++$ and mix gently.

13. Transfer the DMEM++ to two $25-\mathrm{cm}^{2}$ flasks containing subconfluent monolayer cultures of LR7 or FCWF cells, in order to allow propagation of the respective recombinant viruses.

\subsection{Selection and Purification of Recombinant Viruses}

Recombinant MHV or FIPV are selected by growth on LR7 or FCWF cells, respectively. After $24 \mathrm{~h}$ incubation of these cells-overlaid with the infected/transfected cells_at $37^{\circ} \mathrm{C}$, cytopathic effects are visible when recombinant 
wild-type MHV or FIPV is generated. The appearance of cytopathic effects may take more time when preparing viruses with crippling mutations.

1. When cytopathic effects are clearly visible, collect the culture medium and clarify by centrifugation as described above.

2. Store the supernatants at $-80^{\circ} \mathrm{C}$ until the recombinant viruses are purified by two consecutive plaque assays.

3. To this end, autoclave a $3 \%(\mathrm{w} / \mathrm{v})$ agar solution in water and cool it to $43^{\circ} \mathrm{C}$.

4. At the same time, warm the $2 \mathrm{X}$ EMEM concentrate containing FBS and $\mathrm{p} / \mathrm{s}$ at $37^{\circ} \mathrm{C}$.

5. Inoculate cells reaching confluence (LR7 or FCWF cells for MHV or FIPV, respectively) with increasing dilutions in DMEM of the cleared cell culture supernatant.

6. After $1 \mathrm{~h}$ at $37^{\circ} \mathrm{C}$, remove the inoculum and overlay the cells with a $1: 1$ mixture of the agar solution and the 2X EMEM concentrate $\left(2 \mathrm{ml} / 10 \mathrm{~cm}^{2}\right.$ monolayer $)$ (see Note 5).

7. After $5 \mathrm{~min}$ incubation at room temperature, transfer the cells to $37^{\circ} \mathrm{C}$. Typically, plaques are visible after a $24-\mathrm{h}$ incubation.

8. Pick plaques using disposable tips of a $200-\mu 1$ pipette. First, remove the extreme end of the tip using scissors.

9. Subsequently, push the tip through the agar overlay, at the position of a free plaque.

10. Transfer the agar in the tip to $0.5 \mathrm{ml}$ DMEM.

11. Store this at $-80^{\circ} \mathrm{C}$ until it is used in a second round of plaque purification.

12. After the second round of plaque purification, generate a stock of recombinant virus by inoculating a $25-\mathrm{cm}^{2}$ flask containing either LR7 or FCWF cells, using one-half of the purified plaque.

13. Harvest stocks (designated as passage 1) when extensive cytopathic effects are visible.

14. Clear the harvest by centrifugation and store in aliquots at $-80^{\circ} \mathrm{C}$.

\subsection{Verification of Recombinant Phenotype}

1. In general, generate at least two independent recombinants to verify that the observed phenotypic characteristics are the result of the intended genomic modifications.

2. Purify the genomic RNA from the passage 1 stock using the QIAamp Viral RNA mini kit (Qiagen).

3. Genetically analyze the recombinant viruses by reverse transcription-PCR using genomic RNA as a template.

4. Analyze the RT-PCR products using size or restriction fragment analysis and direct sequencing. 


\section{Notes}

1. Unless otherwise stated, prepare all solutions in MilliQ water.

2. Instead of LR7 cells, 17 Cl1, L2, DBT, or Sac(-) cells can be used.

3. Wear gloves and use RNase-free water and materials.

4. Capped synthetic RNA transcripts are used. It is recommended that the synthetic RNA be used within $48 \mathrm{~h}$.

5. Mix by pipetting once up and down; then use immediately. If the temperature of the agar solution used is too high, the cells will be killed. If the temperature is not high enough, the solution will solidify prematurely.

\section{References}

1. Masters, P. S., and Rottier, P. J. (2005) Coronavirus reverse genetics by targeted RNA recombination. Curr. Top. Microbiol. Immunol. 287, 133-159.

2. Koetzner, C. A., et al. (1992) Repair and mutagenesis of the genome of a deletion mutant of the coronavirus mouse hepatitis virus by targeted RNA recombination. $J$. Virol. 66, 1841-1848.

3. van der Most, R. G., et al. (1992) Homologous RNA recombination allows efficient introduction of site-specific mutations into the genome of coronavirus MHV-A59 via synthetic co-replicating RNAs. Nucleic Acids Res. 20, 3375-3381.

4. Masters, P. S., et al. (1994) Optimization of targeted RNA recombination and mapping of a novel nucleocapsid gene mutation in the coronavirus mouse hepatitis virus. J. Virol. 68, 328-337.

5. Godeke, G. J., et al. (2000) Assembly of spikes into coronavirus particles is mediated by the carboxy-terminal domain of the spike protein. J. Virol. 74, 1566-1571.

6. Kuo, L., et al. (2000) Retargeting of coronavirus by substitution of the spike glycoprotein ectodomain: crossing the host cell species barrier. J. Virol. 74, 1393-1406.

7. Haijema, B. J., Volders, H., and Rottier, P. J. (2003). Switching species tropism: an effective way to manipulate the feline coronavirus genome. J. Virol. 77, 4528-38. 